Mathematical Models and Methods in Applied Sciences

Vol. 11, No. 4 (2001) 627-644

(C) World Scientific Publishing Company

\title{
STABILITY OF ABSTRACT LINEAR THERMOELASTIC SYSTEMS WITH MEMORY
}

\author{
CLAUDIO GIORGI* \\ Dipartimento di Matematica, Università di Brescia, \\ via Valotti 9, I-25133 Brescia, Italy \\ VITTORINO PATA ${ }^{\dagger}$ \\ Dipartimento di Matematica "F. Brioschi", Politecnico di Milano, \\ via Bonardi 9, I-20133 Milano, Italy \\ Communicated by N. Bellomo \\ Received 16 April 1999 \\ Revised 10 January 2000
}

\begin{abstract}
An abstract linear thermoelastic system with memory is here considered. Existence, uniqueness, and continuous dependence results are given. In presence of regular and convex memory kernels, the system is shown to be exponentially stable. An application to the Kirchhoff plate equation is given.
\end{abstract}

\section{Introduction}

The aim of this paper is to study the asymptotic behavior in time of an abstract problem related to linear thermoelastic systems with memory. As in classical thermoelasticity, these systems consist of an elastic equation and a heat equation, which are coupled in such a way that the transfer between the mechanical energy and the heat energy is taken into account. Since the elastic behavior has a conservative character, dissipation is allowed by heat energy losses, only. Thermoelastic systems with memory can be viewed as particular models within the theory of linear thermoviscoelasticity, where both thermal and mechanical memory effects occur. ${ }^{14,18,22}$

Accounting for thermal memory effects only, the internal energy of the body depends on both the actual deformation and the past history of the temperature, whereas the heat flux law involves only the past history of the temperature gradient. On the other hand, invariance under time reversal and thermodynamical

*E-mail: giorgi@bsing.ing.unibs.it

†E-mail: pata@mate.polimi.it 
$\operatorname{arguments}^{14}$ allow us to assume the material to have instantaneous response as far as the stress-strain relation is concerned.

In this framework, the thermal dissipation of the body is weaker than in the classical thermoelastic approach, based on the Fourier heat flux law. Moreover, the approach followed here involves perturbation of both the initial data and the past history. As a consequence, the energy decay is much more difficult to detect.

In particular, we are interested in well-posedness and exponential stability of an evolution problem arising in the two-dimensional theory of linear hereditary thermoelasticity. If we consider only small variations of the temperature and its gradient, we may suppose that the following linear system models temperature and vertical displacement evolution of a homogeneous, (thermally and elastically) isotropic Kirchhoff thin plate subject to thermal deformations and hereditary heat conduction law in a two-dimensional domain $\Omega$ :

$$
\begin{aligned}
\partial_{t t} u(t)+\Delta[\Delta u(t)+\vartheta(t)]=0 & \text { in } \Omega \\
\partial_{t} \vartheta(t)+\vartheta(t)-\Delta u_{t}(t)+\int_{0}^{\infty}[\beta(\sigma) \vartheta(t-\sigma)-\kappa(\sigma) \Delta \vartheta(t-\sigma)] d \sigma=0 & \text { in } \Omega
\end{aligned}
$$

for $t \in \mathbb{R}^{+}=(0,+\infty)$, with initial data

$$
\begin{aligned}
u(0) & =u_{0}, \\
\partial_{t} u(0) & =v_{0}, \\
\vartheta(0) & =\vartheta_{0}, \\
\vartheta(-s) & =\vartheta_{0}(s), \quad s \in \mathbb{R}^{+}
\end{aligned}
$$

subject to boundary conditions

$$
\begin{aligned}
u(t)=\Delta u(t)+(1-\lambda) B_{1} u(t)+\vartheta(t) & =0 \quad \text { on } \partial \Omega, \\
\int_{0}^{s} \vartheta(t-y) d y & =0 \quad \text { on } \partial \Omega, \quad s \in \operatorname{Supp}(\kappa)
\end{aligned}
$$

for every $t \in \mathbb{R}^{+}$, with

$$
B_{1} u=2 n_{1} n_{2} \partial_{x_{1} x_{2}} u-n_{1}^{2} \partial_{x_{1} x_{1}} u-n_{2}^{2} \partial_{x_{2} x_{2}} u,
$$

where $x=\left(x_{1}, x_{2}\right), n=\left(n_{1}, n_{2}\right)$ is the unit outward normal to $\partial \Omega$, and $0<\lambda<1 / 2$ is the Poisson ratio. The reader is referred to Sec. 5 for the derivation of the model.

Here, for simplicity, we put all the physical constants, except $\lambda$, equal to one. In addition, the kernels $\beta$ and $\kappa$ are assumed to be bounded convex functions vanishing at $+\infty$.

To formulate system (1.1) in a history space setting, we follow Giorgi, Marzocchi and Pata, ${ }^{5}$ and we introduce a new variable, namely, the summed past history of $\vartheta$ up to time $t$ which is defined by

$$
\eta^{t}(x, s)=\int_{0}^{s} \vartheta(x, t-y) d y=\int_{t-s}^{t} \vartheta(x, y) d y \quad x \in \Omega, s \in \mathbb{R}^{+} .
$$


One can easily check that $\eta$ satisfies the first-order linear evolution equation

$$
\partial_{t} \eta^{t}(s)+\partial_{s} \eta^{t}(s)=\vartheta(t) \quad \text { in } \Omega \times \mathbb{R}^{+}
$$

for $t \in \mathbb{R}^{+}$, along with the boundary condition

$$
\eta^{t}(0)=0 \quad \text { on } \Omega, \forall t \in \mathbb{R}^{+},
$$

and the initial condition

$$
\eta^{0}=\eta_{0} \quad \text { in } \Omega \times \mathbb{R}^{+}
$$

where

$$
\eta_{0}(s)=\int_{0}^{s} \vartheta_{0}(y) d y \quad \text { in } \Omega, s \geq 0
$$

is the initial summed past history of $\vartheta$.

For any $s \in \mathbb{R}^{+}$, we set for simplicity

$$
\mu(s)=-\kappa^{\prime}(s) \quad \text { and } \quad \nu(s)=-\beta^{\prime}(s)
$$

and we assume the following set of hypotheses:

$$
\begin{gathered}
\nu, \mu \in C^{1}\left(\mathbb{R}^{+}\right) \cap L^{1}\left(\mathbb{R}^{+}\right), \\
\nu(s), \mu(s) \geq 0 \quad \forall s \in \mathbb{R}^{+}, \\
\nu^{\prime}(s), \mu^{\prime}(s) \leq 0 \quad \forall s \in \mathbb{R}^{+} .
\end{gathered}
$$

A formal integration by parts yields ${ }^{5}$

$$
\int_{0}^{\infty} \kappa(\sigma) \Delta \vartheta(t-\sigma) d \sigma=\int_{0}^{\infty} \mu(\sigma) \Delta \eta^{t}(\sigma) d \sigma
$$

and

$$
\int_{0}^{\infty} \beta(\sigma) \vartheta(t-\sigma) d \sigma=\int_{0}^{\infty} \nu(\sigma) \eta^{t}(\sigma) d \sigma .
$$

The second relation of (1.3) transforms into (notice that $\operatorname{Supp}(\kappa)=\operatorname{Supp}(\mu)$ )

$$
\eta^{t}(s)=0 \quad \text { on } \partial \Omega \times \operatorname{Supp}(\mu)
$$

for every $t \in \mathbb{R}^{+}$. In view of this choice of variables, we can translate (1.1)-(1.3) into the following initial and boundary value problem.

Problem P. Find the solution $(u, v, \vartheta, \eta)$ to the system

$$
\begin{aligned}
& \partial_{t} u(t)=v(t) \quad \text { in } \Omega, \\
& \partial_{t} v(t)+\Delta[\Delta u(t)+\vartheta(t)]=0 \quad \text { in } \Omega, \\
& \partial_{t} \vartheta(t)+\vartheta(t)-\Delta v(t)+\int_{0}^{\infty} \nu(\sigma) \eta^{t}(\sigma) d \sigma-\int_{0}^{\infty} \mu(\sigma) \Delta \eta^{t}(\sigma) d \sigma=0 \quad \text { in } \Omega, \\
& \partial_{t} \eta^{t}(s)+\partial_{s} \eta^{t}(s)=\vartheta(t) \quad \text { in } \Omega \times \mathbb{R}^{+},
\end{aligned}
$$


for $t \in \mathbb{R}^{+}$, which satisfies the initial and boundary conditions

$$
\begin{aligned}
& u(t)=\Delta u(t)+(1-\lambda) B_{1} u(t)+\vartheta(t)=0 \quad \text { on } \partial \Omega \times \mathbb{R}^{+}, \\
& \eta^{t}(0)=0 \quad \text { on } \Omega \times \mathbb{R}^{+}, \\
& \eta^{t}(s)=0 \quad \text { on } \partial \Omega \times \operatorname{Supp}(\mu) \times \mathbb{R}^{+} \text {, } \\
& u(0)=u_{0} \quad \text { in } \Omega, \\
& v(0)=v_{0} \quad \text { in } \Omega, \\
& \vartheta(0)=\vartheta_{0} \quad \text { in } \Omega \text {, } \\
& \eta^{0}=\eta_{0} \quad \text { in } \Omega \times \mathbb{R}^{+} .
\end{aligned}
$$

Recently, the asymptotic behavior of systems in linear viscoelasticity and thermoviscoelasticity was investigated by many authors. In his pioneering work, ${ }^{3}$ Dafermos considered the thermoelastic problem involving no memory effects, proving the decay of the associated energy for every initial data, for $u, \vartheta$ satisfying Dirichlet-Dirichlet boundary conditions. Since then, much progress has been made to obtain the exponential decay rate of the energy for such equations. In this direction, we quote some works dealing with thermoelasic rods and plates. ${ }^{1,10,11,15-17,19-21,23,25}$

The plan of the paper is as follows. In Sec. 2 we consider a problem in an abstract setting, and we state well-posedness and exponential stability results. Section 3 is devoted to the proof of the theorems. It is worth mentioning that no assumptions on the exponential decay of the memory kernels are needed. In Sec. 4 we apply the abstract results to the plate equation. Finally, in Sec. 5, we present a derivation of the model.

\section{Abstract Setting}

Let $A$ be a strictly positive self-adjoint operator on a Hilbert space $\left(V_{0},\langle\cdot, \cdot\rangle,\|\cdot\|\right)$, of domain $\mathcal{D}(A)=V_{2} \subset V_{0}$, such that $A \in \mathcal{L}\left(V_{2}, V_{0}\right)$ and $A^{-1} \in \mathcal{L}\left(V_{0}, V_{2}\right)$. It is well known that it is possible to define the powers $A^{s}$ of $A$ for $s \in \mathbb{R}$, and the space $V_{s}=\mathcal{D}\left(A^{s / 2}\right)$ turns out to be a Hilbert space with the inner product

$$
\langle\cdot, \cdot\rangle_{s}=\left\langle A^{s / 2} \cdot, A^{s / 2} \cdot\right\rangle \text {. }
$$

We denote by $\|\cdot\|_{s}$ the norm on $V_{s}$ induced by the above inner product (in particular, $\left.\|\cdot\|_{0}=\|\cdot\|\right)$. The injection $V_{s_{1}} \hookrightarrow V_{s_{2}}$ is dense and continuous whenever $s_{1} \geq s_{2}$.

Let $\langle\cdot, \cdot\rangle_{*}$ be another inner product defined on $V_{2}$, which induces a norm $\|\cdot\|_{*}$ which is equivalent to $\|\cdot\|_{2}$. Actually, from the inverse mapping theorem, it would be enough to require that $\|\cdot\|_{*}$ be stronger (or weaker) than $\|\cdot\|_{2}$. This yields the existence of a strictly positive self-adjoint operator $B$ on $V_{0}$ of domain $\mathcal{D}(B)=V_{2}$, $\mathcal{D}\left(B^{s / 2}\right)=V_{s}$ for $s \in \mathbb{R}$, with $B \in \mathcal{L}\left(V_{2}, V_{0}\right)$ and $B^{-1} \in \mathcal{L}\left(V_{0}, V_{2}\right)$, such that

$$
\langle\cdot, \cdot\rangle_{*}=\langle B \cdot, B \cdot\rangle .
$$


Furthermore, $A B^{-1} \in \mathcal{L}\left(V_{0}\right)$ and $\left(A B^{-1}\right)^{-1}=B A^{-1} \in \mathcal{L}\left(V_{0}\right)$. We agree to denote by $V_{*}$ the space $V_{2}$ endowed with the inner product $\langle\cdot, \cdot\rangle_{*}$.

In view of $(\mathrm{h} 1)-(\mathrm{h} 2)$, let

$$
W=L_{\nu}^{2}\left(\mathbb{R}^{+}, V_{0}\right) \cap L_{\mu}^{2}\left(\mathbb{R}^{+}, V_{1}\right)
$$

namely, the Hilbert space of $V_{0}$-valued functions on $\operatorname{Supp}(\nu) \cup \operatorname{Supp}(\mu) \subset \mathbb{R}^{+}$, which are $V_{1}$-valued on $\operatorname{Supp}(\mu)$, endowed with the inner product

$$
\langle\varphi, \psi\rangle_{W}=\int_{0}^{\infty} \nu(\sigma)\langle\varphi(\sigma), \psi(\sigma)\rangle d \sigma+\int_{0}^{\infty} \mu(\sigma)\langle\varphi(\sigma), \psi(\sigma)\rangle_{1} d s
$$

and norm

$$
\|\varphi\|_{W}^{2}=\langle\varphi, \varphi\rangle_{W} .
$$

Finally, we introduce the Hilbert space

$$
\mathcal{H}=V_{*} \times V_{0} \times V_{0} \times W
$$

endowed with the usual inner product.

Setting $z(t)=\left(u(t), v(t), \vartheta(t), \eta^{t}\right)$ and $z_{0}=\left(u_{0}, v_{0}, \vartheta_{0}, \eta_{0}\right) \in \mathcal{H}$, we consider linear evolution equation in $\mathcal{H}$ :

$$
\left\{\begin{array}{l}
\frac{d}{d t} z(t)=L z(t) \\
z(0)=z_{0}
\end{array}\right.
$$

The operator $L$ is defined as

$$
L\left(\begin{array}{c}
u \\
v \\
\vartheta \\
\eta
\end{array}\right)=\left(\begin{array}{c}
v \\
-B\left(B u-\left(A B^{-1}\right)^{*} \vartheta\right) \\
-\vartheta-A v-\int_{0}^{\infty} \nu(\sigma) \eta(\sigma) d \sigma-\int_{0}^{\infty} \mu(\sigma) A \eta(\sigma) d \sigma \\
\vartheta-\partial_{s} \eta
\end{array}\right)
$$

where $\left(A B^{-1}\right)^{*}$ is the adjoint of $A B^{-1}$, with domain

$$
\mathcal{D}(L)=\left\{\begin{array}{l|l}
z \in \mathcal{H} & \begin{array}{l}
B u-\left(A B^{-1}\right)^{*} \vartheta \in V_{*} \\
v \in V_{*} \\
\int_{0}^{\infty} \nu(\sigma) \eta(\sigma) d \sigma+\int_{0}^{\infty} \mu(\sigma) A \eta(\sigma) d \sigma \in V_{0} \\
\vartheta \in W \\
\partial_{s} \eta \in W \\
\eta(0)=0
\end{array}
\end{array}\right\} .
$$

It is worthwhile to notice that, if $\mu \not \equiv 0$ (which means that $L_{\mu}^{2}\left(\mathbb{R}^{+}, V_{1}\right)$ does not reduce to zero), then $\vartheta \in W$ implies $\vartheta \in V_{1}$, and $B u-\left(A B^{-1}\right)^{*} \vartheta \in V_{*}$ implies in turn $u \in V_{3}$. 
Existence, uniqueness and continuous dependence from initial data of the solutions to problem (2.1) is obtained showing that $L$ is the infinitesimal generator of a $C_{0}$-semigroup of bounded linear operators (cf. Pazy ${ }^{24}$ for the definitions).

Theorem 2.1. Assume that the memory kernels $\nu$ and $\mu$ satisfy conditions (h1)(h3). Then $L$ is the infinitesimal generator of a $C_{0}$-semigroup $S(t)=e^{t L}$ of contractions on $\mathcal{H}$, i.e.

$$
\|S(t)\|_{\mathcal{B}(\mathcal{H})} \leq 1 \quad \forall t \geq 0
$$

where $\mathcal{B}(\mathcal{H})$ is the space of bounded linear operators on $\mathcal{H}$.

We recall that a $C_{0}$-semigroup is said to be exponentially stable if there exist two constants $M \geq 1$ and $\varepsilon>0$ such that

$$
\|S(t)\|_{\mathcal{B}(\mathcal{H})} \leq M e^{-\varepsilon t} .
$$

Theorem 2.2. Assume that the memory kernels $\nu$ and $\mu$ satisfy conditions (h1)(h3). Then the semigroup $S(t)$ associated to problem (2.1) is exponentially stable.

\section{Proof of the Theorems}

Proof of Theorem 2.1. We first show that $L$ is dissipative. Indeed, for every $z \in \mathcal{D}(L)$ we have

$$
\begin{aligned}
\langle L z, z\rangle_{\mathcal{H}} & =-\|\vartheta\|^{2}-\left\langle\partial_{s} \eta, \eta\right\rangle_{W} \\
& =-\|\vartheta\|^{2}-\frac{1}{2} \int_{0}^{\infty} \nu(\sigma) \frac{d}{d \sigma}\|\eta(\sigma)\|^{2} d \sigma-\frac{1}{2} \int_{0}^{\infty} \mu(\sigma) \frac{d}{d \sigma}\|\eta(\sigma)\|_{1}^{2} d \sigma \\
& =-\|\vartheta\|^{2}+\frac{1}{2} \int_{0}^{\infty} \nu^{\prime}(\sigma)\|\eta(\sigma)\|^{2} d \sigma+\frac{1}{2} \int_{0}^{\infty} \mu^{\prime}(\sigma)\|\eta(\sigma)\|_{1}^{2} d \sigma \leq 0
\end{aligned}
$$

thanks to (h3). The boundary term of the above integration by parts equals zero, since $\eta(0)=0$ (see Giorgi, Marzocchi and Pata ${ }^{5}$ for justification).

Next, we show that the operator $I-L$ is onto, where $I$ is the identity operator on $\mathcal{H}$. Let $z^{*}=\left(u^{*}, v^{*}, \vartheta^{*}, \eta^{*}\right) \in \mathcal{H}$, and consider the equation

$$
(I-L) z=z^{*}
$$

which, written in components, reads

$$
\begin{aligned}
& u-v=u^{*} \\
& v+B\left(B u-\left(A B^{-1}\right)^{*} \vartheta\right)=v^{*} \\
& 2 \vartheta+A v+\int_{0}^{\infty} \nu(\sigma) \eta(\sigma) d \sigma+\int_{0}^{\infty} \mu(\sigma) A \eta(\sigma) d \sigma=\vartheta^{*} \\
& \eta-\vartheta+\partial_{s} \eta=\eta^{*}
\end{aligned}
$$

Integration of (3.5) with respect to $s$ bears

$$
\eta(s)=\eta(s ; \vartheta)=\left(1-e^{-s}\right) \vartheta+\int_{0}^{s} e^{y-s} \eta^{*}(y) d y .
$$


Substituting (3.2) into (3.3), and (3.2) and (3.6) into (3.4), we obtain the following system:

$$
\left\{\begin{aligned}
u+B\left(B u-\left(A B^{-1}\right)^{*} \vartheta\right) & =u^{*}+v^{*} \\
c_{\nu} \vartheta+c_{\mu} A \vartheta+A u= & \vartheta^{*}+A u^{*}-\int_{0}^{\infty} \nu(\sigma) \int_{0}^{\sigma} e^{y-\sigma} \eta^{*}(y) d y d \sigma \\
& -\int_{0}^{\infty} \mu(\sigma) \int_{0}^{\sigma} e^{y-\sigma} A \eta^{*}(y) d y d \sigma
\end{aligned}\right.
$$

where

$$
c_{\nu}=2+\int_{0}^{\infty} \nu(\sigma)\left(1-e^{-\sigma}\right) d \sigma \quad \text { and } \quad c_{\mu}=\int_{0}^{\infty} \mu(\sigma)\left(1-e^{-\sigma}\right) d \sigma
$$

are non-negative constants in force of (h1)-(h2).

Assume now $c_{\mu}>0$ (corresponding to $\mu \not \equiv 0$ ). It is not hard to check that the right-hand sides of (3.7) belong to $V_{0}$ and $V_{-1}$, respectively. Indeed (see Giorgi, Naso and $\mathrm{Pata}^{7}$ for a similar calculation),

$$
\left\|\int_{0}^{\infty} \mu(\sigma) \int_{0}^{\sigma} e^{y-\sigma} A \eta^{*}(y) d y d \sigma\right\|_{-1} \leq \int_{0}^{\infty} \mu(y)\left\|\eta^{*}(y)\right\|_{1} d y<\infty .
$$

We associate to (3.7) the following bilinear form on $V_{*} \times V_{1}$ :

$$
b((u, \vartheta),(\tilde{u}, \tilde{\vartheta}))=\langle u, \tilde{u}\rangle+\langle u, \tilde{u}\rangle_{*}-\langle\vartheta, \tilde{u}\rangle_{1}+c_{\nu}\langle\vartheta, \tilde{\vartheta}\rangle+c_{\mu}\langle\vartheta, \tilde{\vartheta}\rangle_{1}+\langle u, \tilde{\vartheta}\rangle_{1} .
$$

Hence, by means of Lax-Milgram theorem (see, e.g., Evans ${ }^{4}$ ), the elliptic problem (3.7) admits a unique (weak) solution $(\hat{u}, \hat{\vartheta}) \in V_{*} \times V_{1}$. Thus the vector $\hat{z}=(\hat{u}, \hat{v}, \hat{\vartheta}, \hat{\eta})$, with $\hat{v}=\hat{u}-u^{*}$ and $\hat{\eta}(s)=\eta(s ; \hat{\vartheta})$, solves Eqs. (3.2)-(3.5). Since $\hat{\vartheta} \in V_{1}$, from (3.5) we have that $\hat{\eta} \in W$. From (3.2) we also get that $\hat{v} \in V_{*}$. Hence, comparing (3.4), $\int_{0}^{\infty} \nu(\sigma) \hat{\eta}(\sigma) d \sigma+\int_{0}^{\infty} \mu(\sigma) A \hat{\eta}(\sigma) d \sigma \in V_{0}$, whereas (3.3) entails $A \hat{u}-\hat{\vartheta} \in V_{*}$. Finally, $\partial_{s} \eta \in W$ by comparison in (3.5), and the equality $\hat{\eta}(0)=0$ follows from (3.6). Thus we conclude that $\hat{z} \in \mathcal{D}(L)$.

Concerning the case $c_{\mu}=0$, it is possible to solve the second equation of (3.7) with respect to $\vartheta$, and substitute the result in the first equation, so obtaining

$$
u+B\left(B u+d_{\nu}\left(A B^{-1}\right)^{*} A u\right)=u^{*}+v^{*}+B f
$$

having set $d_{\nu}=1 / c_{\nu}$ and

$$
f=d_{\nu}\left(A B^{-1}\right)^{*}\left(\vartheta^{*}+A u^{*}-\int_{0}^{\infty} \nu(\sigma) \int_{0}^{\sigma} e^{y-\sigma} \eta^{*}(y) d y d \sigma\right) \in V_{0} .
$$

Notice that $u^{*}+v^{*}+B f \in V_{-2}$. The elliptic equation

$$
u+B\left(B u+d_{\nu}\left(A B^{-1}\right)^{*} A u\right)=g
$$

admits a unique (weak) solution in $V_{2}$ whenever $g \in V_{-2}$. Indeed, the associated bilinear form on $V_{*}$ is given by

$$
b(u, \tilde{u})=\langle u, \tilde{u}\rangle+\langle u, \tilde{u}\rangle_{*}+d_{\nu}\langle u, \tilde{u}\rangle_{2} .
$$


We conclude that (3.8) has a solution $\hat{u} \in V_{*}$. Again, the vector $\hat{z}=(\hat{u}, \hat{v}, \hat{\vartheta}, \hat{\eta})$, with $\hat{\vartheta}=-d_{\nu} A \hat{u}+\left(B^{-1} A\right)^{*} f, \hat{v}=\hat{u}-u^{*}$ and $\hat{\eta}(s)=\eta(s ; \hat{\vartheta})$, solves Eqs. (3.2)(3.5). Moreover, comparing (3.5), we get at once that $\hat{\eta} \in W$ (since in this case $\left.W=L_{\nu}^{2}\left(\mathbb{R}^{+}, V_{0}\right)\right)$. Proceeding as in the previous case we complete the other checks.

Since $L$ is dissipative and Range $(\omega I-L)=\mathcal{H}$ for some $\omega>0$, from the classical Lumer-Phillips theorem, ${ }^{24} L$ is the infinitesimal generator of a $C_{0}$-semigroup of contractions $S(t)$.

Proof of Theorem 2.2. In the sequel, we will consider the complexifications of the Hilbert spaces used so far, and the complexification of the operator $L$ (see Giorgi, Naso and $\mathrm{Pata}^{7}$ for more details). To avoid a cumbersome notation, we will keep the same symbol for both $L$ and the Hilbert spaces.

We show that the operator $i \beta-L$ is uniformly bounded below as $\beta \in \mathbb{R} \backslash[-\sigma, \sigma]$ for some $\sigma>0$. This property, in force of a slight generalization of Lemma 2.6 in Giorgi, Naso and $\mathrm{Pata}^{7}$ (see also Curtain and Zwart, ${ }^{2}$ Theorem 5.1.5), assures the exponential decay of the semigroup $S(t)$.

We proceed by contradiction, and assume that the assertion is false. Then there exist sequences $\beta_{n} \in \mathbb{R}$ and $z_{n}=\left(u_{n}, v_{n}, \vartheta_{n}, \eta_{n}\right) \in \mathcal{D}(L)$ with

$$
\left\|z_{n}\right\|_{\mathcal{H}}^{2}=\left\|u_{n}\right\|_{*}^{2}+\left\|v_{n}\right\|^{2}+\left\|\vartheta_{n}\right\|^{2}+\left\|\eta_{n}\right\|_{W}^{2}=1 \quad \forall n \in \mathbb{N}
$$

and

$$
\left|\beta_{n}\right| \geq \sigma \quad \forall n \in \mathbb{N} \quad \text { for some } \sigma>0
$$

such that

$$
\lim _{n \rightarrow \infty}\left\|\left(i \beta_{n}-L\right) z_{n}\right\|_{\mathcal{H}}=0
$$

which is equivalent to

$$
\begin{gathered}
i \beta_{n} u_{n}-v_{n} \underset{n \rightarrow \infty}{\longrightarrow} 0 \text { in } V_{*}, \\
i \beta_{n} v_{n}+B\left(B u_{n}-\left(A B^{-1}\right)^{*} \vartheta_{n}\right) \underset{n \rightarrow \infty}{\longrightarrow} 0 \text { in } V_{0}, \\
i \beta_{n} \vartheta_{n}+\vartheta_{n}+A v_{n}+\int_{0}^{\infty} \nu(\sigma) \eta_{n}(\sigma) d \sigma \\
+\int_{0}^{\infty} \mu(\sigma) A \eta_{n}(\sigma) d \sigma \underset{n \rightarrow \infty}{\longrightarrow} 0 \text { in } V_{0}, \\
i \beta_{n} \eta_{n}-\vartheta_{n}+\partial_{s} \eta_{n} \underset{n \rightarrow \infty}{\longrightarrow} 0 \text { in } W .
\end{gathered}
$$

Repeating the calculations leading to (3.1),

$$
\begin{aligned}
& \left\|\vartheta_{n}\right\|^{2}+\left\langle\partial_{s} \eta_{n}, \eta_{n}\right\rangle_{W} \\
& \quad=\left\|\vartheta_{n}\right\|^{2}-\frac{1}{2} \int_{0}^{\infty} \nu^{\prime}(\sigma)\left\|\eta_{n}(\sigma)\right\|^{2} d \sigma-\frac{1}{2} \int_{0}^{\infty} \mu^{\prime}(\sigma)\left\|\eta_{n}(\sigma)\right\|_{1}^{2} d \sigma
\end{aligned}
$$




$$
\begin{aligned}
& =-\Re\left\langle L z_{n}, z_{n}\right\rangle_{\mathcal{H}} \\
& =\Re\left\langle\left(i \beta_{n}-L\right) z_{n}, z_{n}\right\rangle_{\mathcal{H}} \underset{n \rightarrow \infty}{\longrightarrow} 0 .
\end{aligned}
$$

Therefore

$$
\left\|\vartheta_{n}\right\|^{2} \underset{n \rightarrow \infty}{\longrightarrow} 0
$$

and

$$
\left\langle\partial_{s} \eta_{n}, \eta_{n}\right\rangle_{W} \underset{n \rightarrow \infty}{\longrightarrow} 0
$$

Recall that, from the equivalence of the norms, there exists $C \geq 1$ such that

$$
\frac{1}{C}\|w\|_{*} \leq\|w\|_{2} \leq C\|w\|_{*}, \quad \forall w \in V_{*} .
$$

Thus from (3.10) and (3.11) we get

$$
\frac{1}{\beta_{n}}\left\|v_{n}\right\|_{2} \leq \frac{C}{\beta_{n}}\left\|v_{n}\right\|_{*} \leq K
$$

for some $K>0$ independent of $n \in \mathbb{N}$. The inner product in $V_{0}$ of (3.13) and $\vartheta_{n} / \beta_{n}$ entails

$$
i\left\|\vartheta_{n}\right\|^{2}+\frac{1}{\beta_{n}}\left\|\vartheta_{n}\right\|^{2}+\frac{1}{\beta_{n}}\left\langle A v_{n}, \vartheta_{n}\right\rangle+\frac{1}{\beta_{n}}\left\langle\eta_{n}, \vartheta_{n}\right\rangle_{W} \underset{n \rightarrow \infty}{\longrightarrow} 0 .
$$

Since

$$
\frac{1}{\beta_{n}}\left\langle A v_{n}, \vartheta_{n}\right\rangle \leq \frac{1}{\beta_{n}}\left\|v_{n}\right\|_{2}\left\|\vartheta_{n}\right\| \leq K\left\|\vartheta_{n}\right\| \underset{n \rightarrow \infty}{\longrightarrow} 0,
$$

using (3.15) we conclude from (3.17) that

$$
\frac{1}{\beta_{n}}\left\langle\eta_{n}, \vartheta_{n}\right\rangle_{W} \underset{n \rightarrow \infty}{\longrightarrow} 0
$$

Thus, taking the inner product in $W$ of $(3.14)$ and $\eta_{n} / \beta_{n}$ we are led to

$$
i\left\|\eta_{n}\right\|_{W}^{2}-\frac{1}{\beta_{n}}\left\langle\vartheta_{n}, \eta_{n}\right\rangle_{W}+\frac{1}{\beta_{n}}\left\langle\partial_{s} \eta_{n}, \eta_{n}\right\rangle_{W} \underset{n \rightarrow \infty}{\longrightarrow} 0
$$

and from (3.10), (3.16) and (3.18) we find the relation

$$
\left\|\eta_{n}\right\|_{W}^{2} \underset{n \rightarrow \infty}{\longrightarrow} 0 \text {. }
$$

The inner products in $V_{0}$ of (3.11) and $v_{n}$, and of (3.12) and $u_{n}$, appealing to (3.15), give the two convergences

$$
i \beta_{n}\left\langle u_{n}, v_{n}\right\rangle-\left\|v_{n}\right\|^{2} \underset{n \rightarrow \infty}{\longrightarrow} 0
$$

and

$$
i \beta_{n}\left\langle v_{n}, u_{n}\right\rangle+\left\|u_{n}\right\|_{* \underset{n \rightarrow \infty}{2}}^{2} 0 .
$$

Adding the first relation to the complex conjugate of the second one, we get

$$
\left\|u_{n}\right\|_{*}^{2}-\left\|v_{n}\right\|^{2} \underset{n \rightarrow \infty}{\longrightarrow} 0 \text {. }
$$


On the other hand, from (3.9), (3.15) and (3.19),

$$
\left\|u_{n}\right\|_{*}^{2}+\left\|v_{n}\right\|^{2} \underset{n \rightarrow \infty}{\longrightarrow} 1
$$

and we end up with

$$
\left\|u_{n}\right\|_{*}^{2} \underset{n \rightarrow \infty}{\longrightarrow} \frac{1}{2} \quad \text { and } \quad\left\|v_{n}\right\|^{2} \underset{n \rightarrow \infty}{\longrightarrow} \frac{1}{2} .
$$

We complete the proof finding a contradiction with (3.20). The inner product in $V_{0}$ of (3.12) and $A^{-1} \vartheta_{n}$, yields

$$
i \beta_{n}\left\langle v_{n}, A^{-1} \vartheta_{n}\right\rangle+\left\langle B u_{n}, B A^{-1} \vartheta_{n}\right\rangle-\left\|\vartheta_{n}\right\|^{2} \underset{n \rightarrow \infty}{\longrightarrow} 0
$$

But

$$
\left|\left\langle B u_{n}, B A^{-1} \vartheta_{n}\right\rangle\right| \leq\left\|u_{n}\right\|_{*}\left\|A^{-1} \vartheta_{n}\right\|_{*} \leq C\left\|u_{n}\right\|_{*}\left\|\vartheta_{n}\right\| .
$$

Thus the last two terms of the above relation go to zero, due to (3.15), and we get that

$$
i \beta_{n}\left\langle v_{n}, A^{-1} \vartheta_{n}\right\rangle \underset{n \rightarrow \infty}{\longrightarrow} 0 .
$$

Finally, taking the inner product in $V_{0}$ of (3.13) and $A^{-1} v_{n}$, we obtain

$$
i \beta_{n}\left\langle\vartheta_{n}, A^{-1} v_{n}\right\rangle+\left\langle\vartheta_{n}, A^{-1} v_{n}\right\rangle+\left\|v_{n}\right\|^{2}+\left\langle\eta_{n}, A^{-1} v_{n}\right\rangle_{W} \underset{n \rightarrow \infty}{\longrightarrow} 0
$$

and in force of (3.15) and (3.16), we get

$$
\left\|v_{n}\right\|^{2} \underset{n \rightarrow \infty}{\longrightarrow} 0
$$

which is a contradiction.

\section{Application to the Plate Equation}

Set $V_{0}=L^{2}(\Omega), V_{1}=H_{0}^{1}(\Omega)$ and $V_{2}=H^{2}(\Omega) \cap H_{0}^{1}(\Omega)$, and let $A=-\Delta$, the Laplace operator with Dirichlet boundary condition. On $V_{2}$ introduce the inner product

$$
\begin{aligned}
\langle u, w\rangle_{*}= & \int_{\Omega}\left[\partial_{x_{1} x_{1}} u \partial_{x_{1} x_{1}} w+\partial_{x_{2} x_{2}} u \partial_{x_{2} x_{2}} w+\lambda\left(\partial_{x_{1} x_{1}} u \partial_{x_{2} x_{2}} w+\partial_{x_{2} x_{2}} u \partial_{x_{1} x_{1}} w\right)\right. \\
& \left.+2(1-\lambda) \partial_{x_{1} x_{2}} u \partial_{x_{1} x_{2}} w\right] d x_{1} d x_{2}
\end{aligned}
$$

which defines the strictly positive self-adjoint operator $B$. We recall that for every $u, \vartheta, w$ smooth enough, such that $w=0$ on $\partial \Omega$, the following Green's formula holds $\left(\right.$ see Lagnese $\left.{ }^{12}\right)$ :

$$
\int_{\Omega}\left(\Delta^{2} u\right) w d \Omega=\langle u, w\rangle_{*}-\int_{\partial \Omega}\left[\Delta u+(1-\lambda) B_{1} u\right] \partial_{n} w d(\partial \Omega)
$$

with $B_{1}$ as in (1.4), and

$$
\int_{\Omega}(\Delta \vartheta) w d \Omega=\int_{\Omega}(\Delta w) \vartheta d \Omega-\int_{\partial \Omega} \vartheta \partial_{n} w d(\partial \Omega) .
$$


Hence, exploiting the boundary conditions (1.3), we can give the following:

Definition 4.1. A quadruplet $z=(u, v, \vartheta, \eta) \in C([0,+\infty), \mathcal{H})$ is said to be a variational solution to problem $\mathbf{P}$ if

$$
\begin{aligned}
\left\langle\partial_{t} u, \tilde{u}\right\rangle-\langle v, \tilde{u}\rangle & =0, \\
\left\langle\partial_{t} v, \tilde{v}\right\rangle+\langle u, \tilde{v}\rangle_{*}-\langle\vartheta, A \tilde{v}\rangle & =0, \\
\left\langle\partial_{t} \vartheta, \tilde{\vartheta}\right\rangle+\langle\vartheta, \tilde{\vartheta}\rangle+\langle\vartheta, A \tilde{\vartheta}\rangle+\langle\eta, \tilde{\vartheta}\rangle_{W} & =0, \\
\left\langle\partial_{t} \eta+\partial_{s} \eta, \tilde{\eta}\right\rangle_{W}-\langle\vartheta, \tilde{\eta}\rangle_{W} & =0,
\end{aligned}
$$

for every $\tilde{u} \in V_{0}, \tilde{v} \in V_{2}, \tilde{\vartheta} \in V_{2}, \tilde{\eta} \in W \cap L_{\mu}^{2}\left(\mathbb{R}^{+}, V_{2}\right)$, and almost every $t \in \mathbb{R}^{+}$ (with abuse of notation the brackets are also used to denote duality products); and

$$
\begin{aligned}
u(0)=u_{0} \in V_{2} & \text { a.e. in } \Omega, \\
v(0)=v_{0} \in V_{0} & \text { a.e. in } \Omega, \\
\vartheta(0)=\vartheta_{0} \in V_{0} & \text { a.e. in } \Omega, \\
\eta^{0}=\eta_{0} \in W & \text { a.e. in } \Omega \times \mathbb{R}^{+} .
\end{aligned}
$$

A quick look at (4.1) and (4.2) shows that in fact

$$
\langle A u-\vartheta, A w\rangle=\left\langle B u-\left(A B^{-1}\right)^{*} \vartheta, B w\right\rangle, \quad \forall w \in V_{2} .
$$

Thus, in force of (4.3), the variational solutions to problem $\mathbf{P}$ are given by the semigroup solutions of (2.1), to which, in the hypotheses (h1)-(h3), Theorems 2.1 and 2.2 apply.

\section{The Model Equation}

We consider a thin plate of uniform thickness $d \ll 1$. When the plate is in equilibrium, we assume it occupies a fixed bounded domain $\mathcal{D} \subset \mathbb{R}^{3}$ placed in a reference frame $x=\left(x_{1}, x_{2}, x_{3}\right)$, which has a middle surface midway between its faces in a region $\Omega \subset \mathbb{R}^{2}$ of the plane $x_{3}=0$.

In order to describe the thermomechanical behavior of the plate, we first formulate proper constitutive equations in the framework of the Gurtin-Pipkin's linear theory on heat conduction with memory. ${ }^{9}$ Then, by means of the Kirchhoff strain-displacement relations, we deduce model equations for the plate, as performed in the works of Lagnese. ${ }^{12,13}$ Both approaches rely on the basic assumption that variations of the absolute temperature $\Theta$ are small compared to some uniform reference temperature $\Theta_{0}$, namely

$$
\left|\frac{\Theta-\Theta_{0}}{\Theta_{0}}\right| \ll 1 .
$$

The resulting system models temperature and vertical displacement evolution of a homogeneous, (thermally and elastically) isotropic Kirchhoff plate subject to thermal deformations and hereditary heat conduction law. 
According to Lagnese, ${ }^{13}$ we assume that the plate is composed by an isotropic (mechanically and thermally) linear thermoelastic material. As a consequence, the stress-strain law is given by

$$
\mathbf{S}(x, t)=\mathbb{L}\left[\mathbf{E}(x, t)-\mathbf{E}^{\theta}(x, t)\right],
$$

where the elastic strain $\mathbf{E}$ and the thermal strain $\mathbf{E}^{\theta}$ are second-order tensors, as well as the stress $\mathbf{S}$. The isotropic fourth order tensor

$$
\mathbb{L}=l \mathbf{I} \otimes \mathbf{I}+2 m \mathbb{I}
$$

involves two constants $l$ and $m$ given by

$$
l=\frac{E \lambda}{(1-2 \lambda)(1+\lambda)}, \quad m=\frac{E}{2(1+\lambda)},
$$

where $E>0$ is the Young elastic modulus, and $0<\lambda<1 / 2$ is the Poisson ratio. In small displacement theory, $\mathbf{E}$ is given by

$$
\mathbf{E}(x, t)=\frac{1}{2}\left(\nabla \mathbf{U}(x, t)+(\nabla \mathbf{U})^{\mathrm{T}}(x, t)\right)
$$

where $\mathbf{U}$ is the displacement vector, and

$$
\mathbf{E}^{\theta}(x, t)=\gamma \theta(x, t) \mathbf{I},
$$

where $\theta=\Theta-\Theta_{0}$ denotes the temperature variation, and $\gamma>0$ is the coefficient of thermal expansion. Substitution of the above relation in (5.1) entails

$$
\mathbf{S}(x, t)=\mathbb{L} \mathbf{E}(x, t)-\frac{\gamma E}{1-2 \lambda} \theta(x, t) \mathbf{I} .
$$

As we shall see later, this choice is perfectly compatible with the presence of thermal hereditary terms into the heat flux and internal energy.

On the other hand, following Gurtin and Pipkin, ${ }^{9}$ the linearized constitutive equation of the heat flux vector $\mathbf{q}$ for a thermally isotropic conductor with memory is given by

$$
\mathbf{q}(x, t)=-\int_{0}^{\infty} k(\sigma) \nabla \theta(x, t-\sigma) d \sigma,
$$

where $k: \mathbb{R}^{+} \rightarrow \mathbb{R}$ is the heat flux memory kernel. Unfortunately, here we are not allowed to use the corresponding linearized expression of the internal energy and its balance equation. Indeed, the theory of Gurtin and Pipkin only applies to rigid heat conductor, so that we must resort to some generalization if small deformations are taken into account. Therefore we take advantage of the thermodynamically consistent theory of linear thermoviscoelasticity proposed by Lazzari and Vuk. ${ }^{14}$ There, the usual energy balance equation is replaced by

$$
\rho_{0} h(x, t)=-\nabla \cdot \mathbf{q}(x, t)+\rho_{0} r(x, t),
$$

where $h$ is the thermal power, which denotes the rate of heat absorption per unit of volume, $\rho_{0}>0$ is the density of the medium, and $r$ is the external heat supply per unit of mass. 
As pointed out by Gurtin, ${ }^{8}$ in order to comply the property of invariance under time-reversal the thermal power-strain kernel, accounting for hereditary contributions to mechanical dissipation, must be equal to the stress-temperature kernel, which is involved in a memory-based stress response. Here, because of the instantaneous constitutive relation (5.1), the time-reversal property is automatically fulfilled assuming for $h$ the following linearized constitutive equation:

$$
h(x, t)=\frac{\Theta_{0}}{\rho_{0}} \mathbf{B}: \partial_{t} \mathbf{E}(x, t)+c \partial_{t} \theta(x, t)+\int_{0}^{\infty} a(\sigma) \partial_{t} \theta(x, t-\sigma) d \sigma,
$$

where $\mathbf{B}$ is a symmetric second-order tensor, $c>0$ is the specific heat of the body, and $a: \mathbb{R}^{+} \rightarrow \mathbb{R}$ is the thermal memory kernel. Regarding the kernels $k$ and $a$, we assume that they are smooth enough and summable on $\mathbb{R}^{+}$. Also, we require that $k^{\prime}, a^{\prime \prime}$ are both non-positive, $a(0)>0$, and $k, a^{\prime}$ vanish at infinity.

The particular form of $\mathbf{S}$ and $h$ leads to the lack of memory in the coupling terms of the thermoelastic system (see Giorgi and $\mathrm{Naso}^{6}$ for a more detailed justification of this choice). According to Lazzari and Vuk, ${ }^{14}$ thermodynamic compatibility between (5.1) and (5.6) is fulfilled provided that

$$
\mathbf{B}=\frac{\gamma E}{1-2 \lambda} \mathbf{I}
$$

where $\gamma \neq 0$ is a coupling constant between thermal and mechanical evolution. Without loss of generality $\gamma$ can be taken strictly positive. It is worth observing that if $\gamma=0$, the thermal power $h$ reduces to the time derivative of the GurtinPipkin's internal energy for rigid conductors, and the heat equation (5.5) uncouples from the motion equation.

Henceforth, we denote by $u_{i}\left(x_{1}, x_{2}\right), i=1,2,3$, the components of the displacement vector of the points of the middle surface $\Omega$ of the plate which have coordinates $\left(x_{1}, x_{2}, 0\right)$ at equilibrium. If $d$ is the uniform thickness of the plate, let $\Omega^{-}=\Omega \times\{-d / 2\}$ and $\Omega^{+}=\Omega \times\{d / 2\}$ denote its faces, and $\Gamma=\partial \Omega \times(-d / 2, d / 2)$ its edge, where $\partial \Omega$ is the boundary of $\Omega$.

As customary in thin plate theory, we assume that the transverse normal stress is negligible compared to other stresses, namely $S_{33}=0$. This allows $E_{33}$ to be expressed as a function of $E_{11}$ and $E_{22}$. In addition, integration with respect to $x_{3}$ is carried out, the stretching components $u_{1}, u_{2}$ uncouple from the bending component $u=u_{3}$ of the plate displacement, so that both the strain energy and the kinetic energy split into two parts, accordingly.

Finally, neglecting transverse shear effects, we suppose that $\mathbf{u}=\left(u_{1}, u_{2}, u\right)$ is related to $\mathbf{U}$ by the approximate relations

$$
U_{1}=u_{1}-x_{3} \partial_{x_{1}} u, \quad U_{2}=u_{2}-x_{3} \partial_{x_{2}} u, \quad U_{3}=u .
$$

By virtue of (5.3), this assumption leads to the strain-displacement relations of the 
Kirchhoff model, namely

$$
\left\{\begin{array}{l}
E_{11}=\partial_{x_{1}} u_{1}-x_{3} \partial_{x_{1} x_{1}} u \\
E_{22}=\partial_{x_{2}} u_{2}-x_{3} \partial_{x_{2} x_{2}} u \\
E_{12}=\frac{1}{2}\left[\partial_{x_{2}} u_{1}+\partial_{x_{1}} u_{2}-2 x_{3} \partial_{x_{1} x_{2}} u\right] \\
E_{13}=E_{23}=E_{33}=0
\end{array}\right.
$$

In addition, consistency with the absence of transverse shear requires that the plate be subject to an external distribution of loads per unit of mass $\mathbf{b}=\left(b_{1}, b_{2}, b_{3}\right)$ with $b_{1}$ and $b_{2}$ independent of $x_{3}$.

Introducing the modulus of flexural rigidity

$$
D=\frac{E d^{3}}{12 \rho_{0}\left(1-\lambda^{2}\right)}
$$

and the thermal resultant distribution

$$
\vartheta\left(x_{1}, x_{2}, t\right)=\frac{12}{d^{3}} \int_{-d / 2}^{d / 2} x_{3} \theta\left(x_{1}, x_{2}, x_{3}, t\right) d x_{3}
$$

the motion equation for the bending component of the plate can be obtained exactly as in Lagnese ${ }^{13}$ (see Chap. I, Sec. 6), and reads

$$
d \partial_{t t} u-\frac{d^{3}}{12} \Delta \partial_{t t} u+D \Delta\left[\Delta u+\gamma \frac{1+\lambda}{2} \vartheta\right]=f,
$$

where the dependence on $\left(x_{1}, x_{2}, t\right) \in \Omega \times \mathbb{R}^{+}$is understood and not written, $\Delta$ denotes the two-dimensional Laplacian, and

$$
f\left(x_{1}, x_{2}, t\right)=\int_{-d / 2}^{d / 2} b_{3}\left(x_{1}, x_{2}, x_{3}, t\right) d x_{3} .
$$

Equation (5.10) involves the unknown field $\vartheta$ whose dynamics will be introduced on the basis of the energy balance equation. To this end, we first substitute (5.4), (5.6) and (5.7) into (5.5), to get

$$
\begin{gathered}
\partial_{t} \theta(x, t)+\beta_{0} \mathbf{I}: \partial_{t} \mathbf{E}(x, t)+\int_{0}^{\infty} \alpha(s) \partial_{t} \theta(x, t-\sigma) d \sigma \\
-\int_{0}^{\infty} \kappa(\sigma) \Delta_{3} \theta(x, t-\sigma) d \sigma=\frac{1}{c} r(x, t)
\end{gathered}
$$

where $\Delta_{3}$ denotes the three-dimensional Laplacian, and

$$
\beta_{0}=\frac{\Theta_{0} \gamma E}{\rho_{0} c(1-2 \lambda)}, \quad \kappa(s)=\frac{k(s)}{\rho_{0} c}, \quad \alpha(s)=\frac{a(s)}{c} .
$$

Then we insert relations (5.8) of the Kirchhoff model into (5.11); we multiply the result by $12 x_{3} / d^{3}$ and integrate in $x_{3}$ from $-d / 2$ to $d / 2$. Recalling (5.9), and setting

$$
g\left(x_{1}, x_{2}, t\right)=\frac{12}{d^{3} c} \int_{-d / 2}^{d / 2} x_{3} r\left(x_{1}, x_{2}, x_{3}, t\right) d x_{3}
$$


and

$$
p\left(x_{1}, x_{2}, t\right)=\frac{12}{d^{3}} \int_{-d / 2}^{d / 2} x_{3}\left[\int_{0}^{\infty} \kappa(\sigma) \partial_{x_{3} x_{3}} \theta\left(x_{1}, x_{2}, x_{3}, t-\sigma\right) d \sigma\right] d x_{3}
$$

we end up with

$$
\begin{aligned}
\partial_{t} \vartheta(t) & -\beta_{0} \Delta \partial_{t} u(t)+\int_{0}^{\infty} \alpha(\sigma) \partial_{t} \vartheta(t-\sigma) d \sigma \\
& -\int_{0}^{\infty} \kappa(\sigma) \Delta \vartheta(t-\sigma) d \sigma=g(t)+p(t),
\end{aligned}
$$

where the dependence on $\left(x_{1}, x_{2}\right)$ is understood. Now, integration by parts with respect to $x_{3}$ yields

$$
\begin{aligned}
p(t) & =\left.\frac{12}{d^{3}} \int_{0}^{\infty} \kappa(\sigma)\left(x_{3} \partial_{x_{3}} \theta(t-\sigma)-\theta(t-\sigma)\right)\right|_{x_{3}=-d / 2} ^{x_{3}=d / 2} d \sigma \\
& =\frac{6}{d^{2}}\left(\left.\mathbf{q} \cdot \mathbf{n}\right|_{\Omega^{+}}-\left.\mathbf{q} \cdot \mathbf{n}\right|_{\Omega^{-}}\right)-\left.\frac{12}{d^{3}} \int_{0}^{\infty} \kappa(\sigma) \theta(t-\sigma)\right|_{x_{3}=-d / 2} ^{x_{3}=d / 2} d \sigma
\end{aligned}
$$

where $\left.\mathbf{n}\right|_{\Omega^{-}}$and $\left.\mathbf{n}\right|_{\Omega^{+}}$denote the unit outward normals to the lower and upper faces of the plate, respectively. Concerning the first term of the right-hand side of the above relation, a quite natural boundary condition for the plate is that the heat flux vanishes across its faces, namely

$$
\left.\mathbf{q} \cdot \mathbf{n}\right|_{\Omega^{+}}=\left.\mathbf{q} \cdot \mathbf{n}\right|_{\Omega^{-}}=0 .
$$

In order to evaluate the second term, for every $x_{3} \in(-d / 2, d / 2)$ and $\left(x_{1}, x_{2}\right) \in \Omega$ we make the following assumption (which is justified because of the thinness of the plate):

$$
\theta\left(x_{1}, x_{2}, x_{3}, t\right)=\vartheta_{0}\left(x_{1}, x_{2}, t\right)+\vartheta_{1}\left(x_{1}, x_{2}, t\right) x_{3}+\vartheta_{2}\left(x_{1}, x_{2}, t\right) x_{3}^{2}+o\left(d^{3}\right) .
$$

In force of (5.9) it follows that

$$
\vartheta_{1}=\vartheta+o(d) \quad \text { and }\left.\quad \theta\right|_{x_{3}=-d / 2} ^{x_{3}=-d / 2}=d \vartheta+o\left(d^{3}\right)
$$

Hence, (5.13) and (5.14) lead to the approximate equality

$$
p(t)=-\frac{12}{d^{2}} \int_{0}^{\infty} \kappa(\sigma) \vartheta(t-\sigma) d \sigma .
$$

A similar result can be obtained assuming a uniform environmental temperature and applying Newton's law of cooling instead of adiabatic boundary condition on the lower and upper faces (see, for instance, Lagnese, ${ }^{13}$ p. 30). As a consequence, (5.12) becomes

$$
\begin{aligned}
\partial_{t} \vartheta(t) & -\beta_{0} \Delta \partial_{t} u(t)+\int_{0}^{\infty} \alpha(\sigma) \partial_{t} \vartheta(t-\sigma) d \sigma-\int_{0}^{\infty} \kappa(\sigma) \Delta \vartheta(t-\sigma) d \sigma \\
& +\frac{12}{d^{2}} \int_{0}^{\infty} \kappa(\sigma) \vartheta(t-\sigma) d \sigma=g(t)
\end{aligned}
$$


According to the assumed regularity of kernel $\alpha$, we observe that

$$
\int_{0}^{\infty} \alpha(\sigma) \partial_{t} \vartheta(t-\sigma) d \sigma=\alpha(0) \vartheta(t)+\int_{0}^{\infty} \alpha^{\prime}(\sigma) \vartheta(t-\sigma) d \sigma
$$

and substitution into (5.15) bears

$$
\begin{aligned}
\partial_{t} \vartheta(t) & +\alpha_{0} \vartheta(t)-\beta_{0} \Delta \partial_{t} u(t) \\
& +\int_{0}^{\infty}[\beta(\sigma) \vartheta(t-\sigma)-\kappa(\sigma) \Delta \vartheta(t-\sigma)] d \sigma=g(t)
\end{aligned}
$$

where

$$
\beta(s)=\alpha^{\prime}(s)+\frac{12}{d^{2}} \kappa(s) \quad \text { and } \quad \alpha_{0}=\alpha(0)>0 .
$$

Finally, we turn our attention to the boundary conditions. If the plate is simply supported along its edge $\Gamma$, then the corresponding boundary conditions on $u$ and $\vartheta$ along $\partial \Omega$ are as follows (see Lagnese, ${ }^{13} \mathrm{Eq} .(2.20)$ )

$$
u(t)=\Delta u(t)+(1-\lambda) B_{1} u(t)+\gamma \frac{1+\lambda}{2} \vartheta(t)=0
$$

with $B_{1}$ as in (1.4).

In order to derive a boundary condition relative to the thermal environment, we assume the plate to reside in a medium of uniform temperature (measured from the reference temperature). Accounting for hereditary conductors, and assuming that the environmental temperature equals the reference one, Newton's law of cooling can be generalized as follows:

$$
k(y) \partial_{n} \theta(t-y)=-\lambda_{0} \theta(t-y) \quad \text { on } \Gamma
$$

for almost every $y \in \operatorname{Supp}(k)$, where $\lambda_{0} \geq 0$ is the conductivity constant of the edge of the plate, and $\partial_{n}$ denotes the derivative along the outward unit normal $n$ introduced in (1.4). Clearly, $\operatorname{Supp}(k)=\operatorname{Supp}(\kappa)$. Multiplying this relation times $12 x_{3} /\left(\rho_{0} d^{3} c\right)$, and integrating with respect to $x_{3}$ over $[-d / 2, d / 2]$, we get, in view of (5.9),

$$
\kappa(y) \partial_{n} \vartheta(t-y)=-\frac{\lambda_{0}}{\rho_{0} c} \vartheta(t-y) \quad \text { on } \partial \Omega, \quad \text { a.e. } y \in \operatorname{Supp}(\kappa) .
$$

Observing that if $s \in \operatorname{Supp}(\kappa)$ then $[0, s] \subset \operatorname{Supp}(\kappa)$, we can integrate over $[0, s]$ to obtain

$$
-\frac{\rho_{0} c}{\lambda_{0}} \int_{0}^{s} \kappa(y) \partial_{n} \vartheta(t-y) d y=\int_{0}^{s} \vartheta(t-y) d y \quad \text { on } \partial \Omega, \quad s \in \operatorname{Supp}(\kappa) .
$$

Finally, letting $\lambda_{0} \rightarrow \infty$, i.e. assuming that the edge has infinite thermal conductivity, we conclude that

$$
\int_{0}^{s} \vartheta(t-y) d y=0 \quad \text { on } \partial \Omega, \quad s \in \operatorname{Supp}(\kappa) .
$$


Collecting Eqs. (5.10) (neglecting rotatory inertia, namely, taking the term $d^{3} \Delta \partial_{t t} u / 12$ equal to zero) and (5.16)-(5.18), we are led to the following boundary value problem, which resumes the thermomechanical evolution of the plate:

$$
\begin{aligned}
& d \partial_{t t} u(t)+D \Delta\left[\Delta u(t)+\gamma \frac{1+\lambda}{2} \vartheta(t)\right]=f(t) \quad \text { in } \Omega \\
& \begin{aligned}
\partial_{t} \vartheta(t) & +\alpha_{0} \vartheta(t)-\beta_{0} \Delta \partial_{t} u(t) \\
& +\int_{0}^{\infty}[\beta(\sigma) \vartheta(t-\sigma)-\kappa(\sigma) \Delta \vartheta(t-\sigma)] d \sigma=g(t) \quad \text { in } \Omega,
\end{aligned} \\
& u(t)=\Delta u(t)+(1-\lambda) B_{1} u(t)+\gamma \frac{1+\lambda}{2} \vartheta(t)=0 \quad \text { on } \partial \Omega, \\
& \int_{0}^{s} \vartheta(t-y) d y=0 \quad \text { on } \partial \Omega, \quad s \in \operatorname{Supp}(\kappa) .
\end{aligned}
$$

Clearly, if no external forces and heat supplies are applied to the plate, the functions $f$ and $g$ vanish.

Remark 5.1. When smooth kernels $\mu$ and $\nu$ are involved, the resulting system describes heat propagation with a thermal damping weaker than in the classical approach based on the Fourier law. However, some additional thermal dissipation is supported by the hereditary term into the constitutive Eq. (5.5) for the thermal power $h$. This term, and especially $\alpha(0)$, plays a crucial role in our main stability result. From a physical point of view, it compensates the absence of heat outflow due to the adiabatic boundary condition on the lower and upper faces. This is why we conjecture that exponential stability of the plate fails to hold when the memory term is neglected in the expression of $h$.

\section{Acknowledgment}

This work has been partially supported by the Italian MURST '98 Research Projects "Mathematical Models for Materials Science" and "Problems and Methods in the Theory of Hyperbolic Equations".

\section{References}

1. J. A. Burns, Z. Liu and S. Zheng, On the energy decay of a linear thermoelastic bar, J. Math. Anal. Appl. 179 (1993) 574-591.

2. R. F. Curtain and H. J. Zwart, An Introduction to Infinite-Dimensional Linear System Theory (Springer, 1995).

3. C. M. Dafermos, On the existence and the asymptotic stability of solution to the equations of linear thermoelasticity, Arch. Rational Mech. Anal. 29 (1968) 241-271.

4. L. C. Evans, Partial Differential Equations (Amer. Math. Soc., 1998).

5. C. Giorgi, A. Marzocchi and V. Pata, Asymptotic behavior of a semilinear problem in heat conduction with memory, NoDEA Nonlinear Differential Equations Appl. 5 (1998) 333-354. 
6. C. Giorgi and M. G. Naso, Mathematical models of thin thermo-viscoelastic plates, Quart. J. Mech. Appl. Math. 53 (2000) 363-374.

7. C. Giorgi, M. G. Naso and V. Pata, Exponential stability in linear heat conduction with memory: a semigroup approach, Comm. Appl. Math. 5 (2001) 121-134.

8. M. E. Gurtin, Time-reversal and symmetry in the thermodynamics of materials with memory, Arch. Rational Mech. Anal. 44 (1971/72) 387-399.

9. M. E. Gurtin and A. C. Pipkin, A general theory of heat conduction with finite wave speeds, Arch. Rational Mech. Anal. 31 (1968) 113-126.

10. S. W. Hansen, Exponential energy decay in a linear thermoelastic rod, J. Math. Anal. Appl. 167 (1992) 429-442.

11. J. U. Kim, On the energy decay of a linear thermoelastic bar and plate, SIAM J. Math. Anal. 23 (1992) 889-899.

12. J. Lagnese, Boundary Stabilization of Thin Plates, SIAM Stud. Appl. Math., No. 10 (SIAM, 1989).

13. J. Lagnese and J. L. Lions, Modelling Analysis and Control of Thin Plates (Masson, 1988).

14. B. Lazzari and E. Vuk, Constitutive equations and quasi-static problem in linear thermoviscoelasticity, Internat. J. Engrg. Sci. 30 (1992) 533-544.

15. K. Liu and Z. Liu, Exponential stability and analiticity of abstract linear thermoelastic systems, Z. Angew. Math. Phys. 48 (1997) 885-904.

16. Z. Liu and S. Zheng, Exponential stability of the semigroup associated with a thermoelastic system, Quart. Appl. Math. 51 (1993) 535-545.

17. Z. Liu and S. Zheng, On the exponential stability of linear viscoelasticity and thermoviscoelasticity, Quart. Appl. Math. 54 (1996) 21-31.

18. M. McCarthy, Constitutive equations for thermo-mechanical materials with memory, Internat. J. Engrg. Sci. 8 (1970) 467-474.

19. J. E. Muñoz Rivera, Energy decay rate in linear thermoelasticity, Funkcial. Ekvac. 35 (1992) 19-30.

20. J. E. Muñoz Rivera, Decomposition of the displacement vector field and decay rates in linear thermoelasticity, SIAM J. Math. Anal. 24 (1993) 390-406.

21. J. E. Muñoz Rivera, Asymptotic behaviour in inhomogeneous linear thermoelasticity, Appl. Anal. 53 (1994) 55-65.

22. C. B. Navarro, Asymptotic stability in linear thermoviscoelasticity, J. Math. Anal. Appl. 65 (1978) 399-431.

23. V. Pata and E. Vuk, On the exponential stability of linear thermoelasticity, Continuum Mech. Thermodyn. 12 (2000) 121-130.

24. A. Pazy, Semigroups of Linear Operators and Applications to Partial Differential Equations (Springer-Verlag, 1983).

25. M. Slemrod, Global existence, uniqueness, and asymptotic stability of classical smooth solutions in one-dimensional nonlinear thermoelasticity, Arch. Rational Mech. Anal. 76 (1981) 97-133. 